\title{
Integration of 3D Data and Text: The Effects of Text Positioning, Connectivity, and Visual Hints on Comprehension
}

\author{
Henry Sonnet ${ }^{1}$, Sheelagh Carpendale ${ }^{2}$, and Thomas Strothotte ${ }^{1}$ \\ 1 University of Magdeburg, Dept. of Simulation and Graphics, \\ D-39016 Magdeburg, Germany \\ 2 University of Calgary, Dept. of Computer Science, \\ Calgary, Alberta, Canada T2N 1N4
}

\begin{abstract}
D computer graphic models hold much promise as illustrations that can be interactively explored. These 3D illustrations often need to be linked to labels, annotations and sometimes more lengthy textual explanations. Achieving effective integration of the 3D illustration and its textual information is a difficult task and has resulted in a variety of proposed approaches. However, the comparative effectiveness of these approaches has not been studied. To address this issue, we have conducted a study in which we have compared methods of associating text with its 3D model: attaching the text directly to the object, placing the text in the object's shadow, using symbols to make the correlation between the object and the text, and using a line to make the visual connection from the text to the object with and without additional hints in the shadow. During the first part we were interested in whether a graphical method can clarify the correlation between a part of the 3D model and its associated text. The second part focused on whether the text remains comprehensible during a scene exploration. Based on our results, we suggest design implications for developing interactive 3D illustrations.
\end{abstract}

\section{Introduction}

Data for 3D models exists in a large number of domains such as medicine, automotive engineering, and architecture. Often, it is accompanied by textual information that explains the components of the model or provides further information. In this regard, BRINKLEY et al. [1] distinguish between spatial information (e.g., images, 3D models, or animations) and symbolic information (metadata about the images and 3D models). They state that both types of information are of equal importance in understanding the data presented.

In 3D illustrations, the two distinct types of information, 3D data and text, need to be arranged in a single layout. According to LOK and FEINER [2], the term layout refers to the process of determining each visual object's position and size. FEKETE and PLAISANT [3] have identified a number of basic principles concerning an adequate layout. They state that text should be readable, non-ambiguously related to its graphical object, and not hide any other pertinent information. In order to follow those principles in illustrations that allow for interactive scene exploration (dynamic layouts), the textual 
information continuously needs to be adapted (e. g., position, size, text layout) according to the current view of the data. Several techniques for an interactive information exploration of 3D models and their textual annotations have been proposed.

In most applications, image or model components and their associated labels are connected with lines (e. g., [45]). Additional relational data can be provided in context menus [6]. It can also be accessed in separate areas, for instance, a user can browse through extensive textual description that explains an explorable 3D model, in a separate window [7]. Alternatively, instead of directly integrating the words into a scene, annotation markers can serve as a reference to the textual information (e.g., [89]). There are also a number of dynamic techniques, in which labels move, appear, or disappear during interaction (e. g., [3[10]). In other approaches, an alternative representation of the original scene is used to provide additional explanation (e.g.,[11|12[13]). Here, scene components are projected onto a plane in order to be highlighted and annotated in the background without disturbing the view of the scene. SONNET et al. [14] have proposed a technique in which annotations are directly connected to their corresponding scene objects.
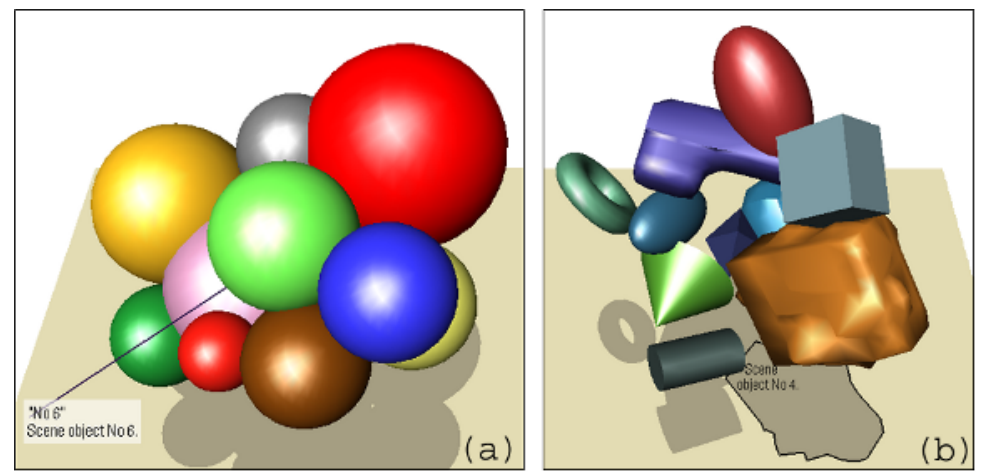

Fig. 1. Two examples of 3D scene and text integration

We were interested in gaining a better understanding of the usability of these types of techniques. In particular we were interested in the clarity of the correlation between the object in the 3D model and its associated text, the effect of including additional visual hints, and whether the text remains readable and comprehensible during scene exploration. Figure 1 shows examples of two different techniques for linking text with objects in a 3D scene. A common method, linking text and its associated object with a line, is shown in Fig. 1. Not. Notice how this scene is also an example of an unclear correlation between object and text. Is the green, red, or another sphere referenced? Figure 1 (b) shows a method of augmenting shadows to provide information. The effectiveness of these types of techniques has not been studied.

In this paper, we present a user study that has been designed to explore the issues involved when integrating 3D data and textual information. During the study, participants were given tasks that involved the exploration of 3D scenes. These tasks included 
finding the correct object-text correlations and following 3D scene exploration instructions that where embedded in the 3D scene. All of the 3D models were composed of arbitrary geometric objects. Data was collected through recording participant activities, questionnaires and the participant's comments and opinions.

This paper is organized as follows: In Section 2, we describe the purpose of our study. Basic aspects regarding the study are given in Section 3 Section 4 contains the individual tasks and designs. Results of the study are included in Section 5 In Section 6 these results are discussed before Section 7 summarizes the evaluation.

\section{Study Goals}

Static layouts can be more easily designed than dynamic layouts. In a static layout, text labels can be positioned carefully so that they do not occlude relevant parts of the image and so that it is clear which image parts are referenced by which labels. But when a static layout is turned into a dynamic layout, the formerly clear layout may become confusing. Image parts may now be located at new positions while associated texts have not been transformed appropriately. As mentioned above, while several techniques have been proposed with the intention of creating adequate dynamic layouts, it is not clear to what extent they achieve these goals. For example, a 3D scene that is supplemented by text may be complex, in that it may have many interleaved components. In a complex scene like this, creating a useful text integration is already complicated in static layouts, let alone dynamic layouts. In particular, we were interested in:

- the effect of text integration techniques and scene complexity on people's ability to correctly correlate a scene object with its associated text,

- the effect of including the additional hint of highlighting an object's shadow on people's ability to correctly correlate a scene object with its associated text, and

- the readability and comprehension of presented text.

\section{Study Method}

\subsection{Participants and Setup}

Thirty-six voluntary participants (10 female / 26 male), aged between 16 and 32, were recruited from the local computer science department. All of the participants were familiar with mouse navigation and $11 \%$ of them had no or little experience with 3D interaction, $25 \%$ had some experience navigating 3D scenes, and $64 \%$ had considerable experience in $3 \mathrm{D}$ navigation from playing 3D computer games or using 3D modeling software.

All tasks were performed at a SGI Octane Workstation with a 21" display. The software used for all tasks differed only in the method used to correlate the textual information with the objects in the 3D scene and in whether or not additional visual hints were included. All the environments allow for interactive exploration of 3D scenes. By exploration we mean the capability to view the scene from arbitrary viewpoints while acquiring information from texts. There were no differences in the techniques that enabled a user to spatially explore the 3D scenes, that is, all other interactions, rotations, 
etc. were identical. In the software, each 3D scene has a model, which is a 3D arrangement of 3D geometric objects of different colors and a setting, which is the manner by which the textual information is correlated to the objects in the model.

\subsection{Description of the Settings and Models}

We chose three different types of settings to evaluate. Setting I was chosen because it integrates the text into the 3D scene. It is an example of the commonly used object-linelabel technique, except that the line is replaced by a translucent polygon. Setting II was chosen because it represents an unusual text integration approach and Setting III was chosen because it represents the common separation between text and 3D model. These three settings were used for Tasks 1 and 3 (see Sect.4).

- Setting I-Object-attached labels. Annotations are directly connected to their respective scene objects in Setting I. As can be seen in Fig. 2(a), a translucent polygon, intersecting with the scene object, provides a visual link between annotation and object. During scene exploration, annotations remain attached to their associated scene objects. The annotations' size is adapted to their scene objects' bounding box sizes, which gives additional hints regarding connectivity. A detailed description of this technique can be found in [14].

- Setting II-Shadow annotations. In Setting II, annotations are located within a shadow plane [13]. The annotations are bordered by the outlines of the respective scene objects after they have been projected onto this plane (see Fig. 2(b)). Since annotations may be occluded by scene objects, they can be temporarily displayed in the foreground on user request. Also, since the available space for text strongly depends on the object projections' shapes, text may not entirely fit into its assigned space. The available space may only be enlarged by zooming into the scene.

- Setting III-Separated annotations. In contrast to the previous settings where annotations are integrated within the same window as the 3D scene, in Setting III text is located in a separate window. As can be seen in Fig.2(c), the textual information is shown within the windows on the right hand side. In order to identify individual objects, a clear textual description of all the scene objects is provided.
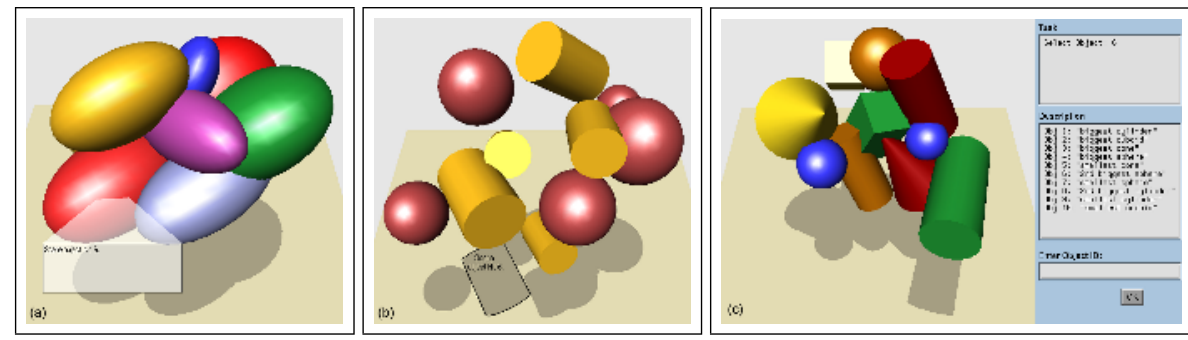

Fig. 2. (a) Setting I (Model 1): annotations are directly attached to scene objects using translucent polygonal shapes. (b) Setting II (Model 2): annotations are located within the objects' shadows in the scene. (c) Setting III (Model 3): area showing the 3D model and text area are separated. Textual information-including scene object descriptions-is displayed in the window on the right hand side. 
For Task 2 we explored whether what is perhaps the most common method of labelingattaching the text to its associated object by means of a simple line-can be improved with the addition of visual hints. From our own experience and from pilot studies we knew that this method could be ambiguous. Therefore for Task 2, the settings, A, B and $\mathrm{C}$, vary in regards to the type of visual hint used.
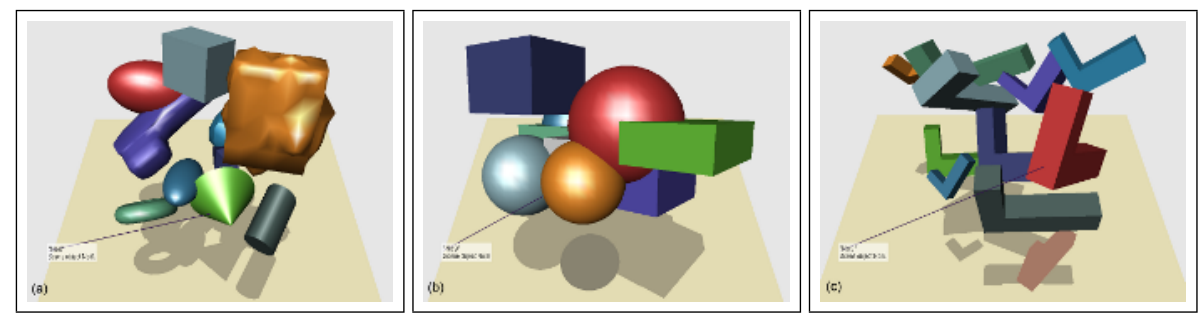

Fig. 3. All these settings use a simple line and text label annotation technique: (a) Setting A, (Model 4) makes use of no additional hints, (b) Setting B, (Model 5) the shadow of the object is highlighted using a gray tone, and (c) Setting C, (Model 6) the highlighting uses the diffuse color of the respective scene object

- Setting A. Object and text are only linked with a line. There are no further hints that may help to find a correlation between scene object and annotation (see Fig. 3 a)). The text remains at a static position during scene exploration. Only the line end that is connected to a point inside the corresponding scene object moves with the object.

- Setting $B$. Beside the line that connects scene object and annotation, the respective projection of the annotated scene object (object's shadow) is highlighted using a gray value that differs from the general shadow color (see Fig. 3(b)).

- Setting $C$. The only difference to Setting $B$ is that the annotated object's shadow is highlighted using the diffuse color of the corresponding scene object. To this end, the shadow color as well as the position of the shadow relative to the respective scene object can be used to locate the annotated scene object (see Fig. 3. (c)).

The models used during the study were composed of arbitrary geometric objects. Basically, there were two reasons for why we decided to use arbitrary models: (1) to prevent previous knowledge from aiding some participants when objects were to be found on the basis of descriptions and (2) each model was constructed to consciously stress specific aspects to be analyzed during the study. For example, we were interested in the different effects of compact models and models whose objects were arranged more apart. Or what would be the difference between models composed of similarly and variously shaped objects?

\section{The Tasks}

Each participant performed three tasks and each task had three different settings. So that familiarity with the 3D model did not affect the results, for each setting, one of 
three different models was used. The order in which the participants saw the settings and which model was used with which setting was counterbalanced. Before each task, participants were given a careful explanation of the task and were given practice runs. In addition to these interaction tasks, participants were asked to complete three questionnaires: at the beginning, the end, and after completing Task 1. The participants were also asked if they had any comments they would like to make.

Participants were told that their actions would be recorded. This included the time taken for each task, which objects were selected and text annotations that were preformed. They were also told that it was more important to solve the tasks correctly rather than to be quick. The participants were observed during the tasks and notes were taken about their actions and comments.

Problems that may occur when integrating textual information into a scene include: unclear correlation between scene object and text, scene occlusions, or other interferences during scene exploration. The following three tasks were designed to explore these issues.

\subsection{Task 1: Object-Text Correlations}

Using Settings I-III and three different 3D models, participants were asked to find the correlations between the scene objects and the annotations. Specifically a participant was asked to identify the scene object that the annotation currently displayed referred to, by selecting that object. For that purpose, eight annotations were shown for each setting (see Fig. 2). A new annotation appeared every time the participant had completed the subtask of selecting a scene object. In Settings I and II, the annotations were displayed together with the rendered model within the same window. Setting III differed from the other settings in that scene object descriptions were shown in a window on the right hand side. Instead of eight annotations integrated into the scene, successively the descriptions of eight objects were shown in the annotation window. The participant found the objects being referred to by their descriptions (see Fig. 2 ( c)). Prior to each recorded study run a practice run with a test model was performed. The two attributes Setting and $3 D$ model have been varied such that each participant performed the task with Settings I-III and three different models.

\subsection{Task 2: Object-Text Correlations with Additional Hints}

This task was designed to discover whether there are benefits to be gained when additional hints are included with simple labels attached by straight lines. The task used Settings A-C, see Fig. 3. We expected that the ancillary hints would help a user to find correct object-text correlations.

The basic task was the same as Task 1. Participants were asked to find the correlations between the scene objects and the annotations. Again, successively eight annotations appeared that should be assigned to the scene objects. The 3D models differed from those of Task 1 as can be seen in Fig. 3. The setting-model pairs were varied such that no participant performed the task with the same model twice.

\subsection{Task 3: Task-Based Scene Exploration}

This task was designed to explore reading and understanding the displayed text information and this was followed by a task-based scene exploration. The task itself was 
displayed to the participant in the text annotation of the setting that was in use. The participants were asked to read the text and then to follow the instructions it contained. There were two aspects we addressed with this part of the study: Can the participants gather the provided information properly and are there potential interferences between 3D model and text during scene exploration?
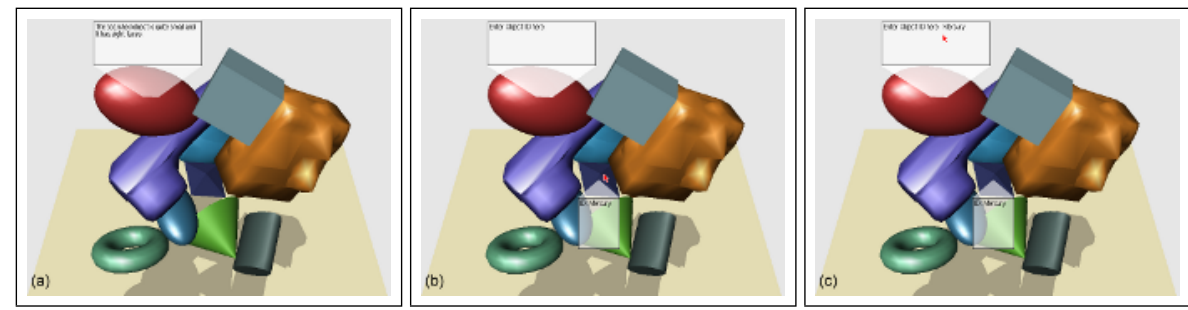

Fig. 4. Task 3: Setting I (Model 4). (a) The description of the scene object that is to be found is displayed attached to a different scene object. The participant uses this information to find and select the described scene object; on selection a second annotation that contains the object ID appears (b). Finally, this object ID is then appended to the first annotation (c).

The task had three components:

1. Read the complete information given by a displayed text.

2. Find the scene object according to the information gathered from the displayed text.

3. Append an ID—associated with the found scene object— to the displayed text.

With component (1) we wanted to find out if participants would have problems when reading the complete displayed information. Whether the participants understand the information could be observed by how they carried out the instructions for the second part of the task (2). Carrying out component (3) was included to shed some light on possible interferences between 3D model and text such as model/text occlusions that might occur during the search for the described objects with their IDs and during the annotation of the text information with this ID. Figure 4 illustrates this task.

Task 3 proceeded as follows: After an annotation-attached to an arbitrary scene object-was displayed (see Figs. 4(a) or 5(a)), the participant read the complete text. Note that the textual description referred to a different scene object from the object that the annotation was attached to. When the participant found and selected the described scene object, an annotation with the found object's ID appeared (see Figs. 4 (b) or 5 (b)). At this point both annotations were integrated into the scene and remained visible during interaction. The last step was to enter the found object's ID into the first annotation. When this annotation had been selected, its new text was "Enter Object ID here:" instead of the task description (see Figs. 4(c) or 5(c)). This procedure was repeated 5 times each for Settings I and II. In Setting III, annotations were not integrated into the render area. Hence, the procedure differed from Settings I and II (see Fig. 6) in that readings were preformed in the side window. 

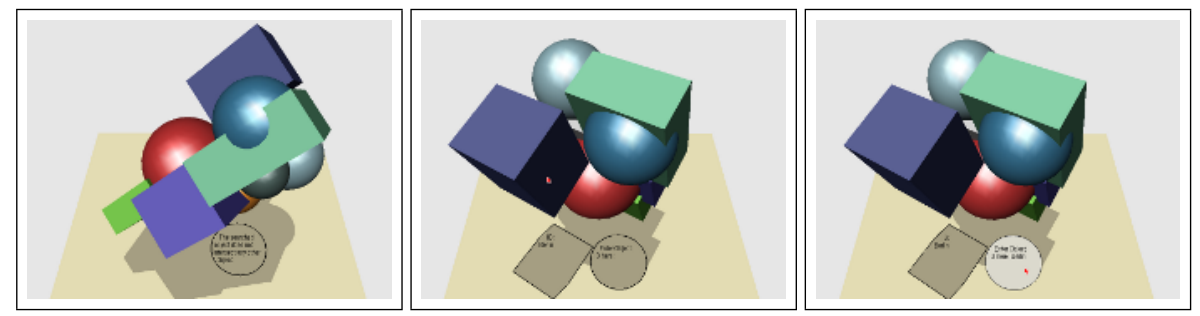

Fig. 5. Task 3: Setting II (Model 5). The same procedure as in Fig. 4 with the difference that all text is displayed within the shadow plane.
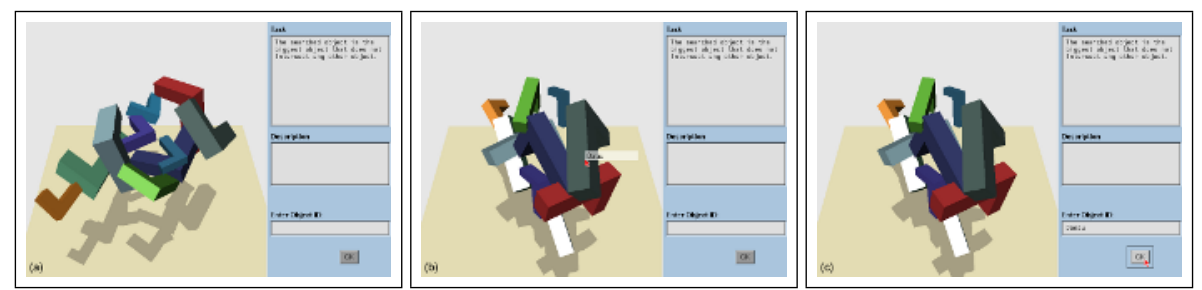

Fig. 6. Task 3: Setting III (Model 6). (a) The scene object's description is shown in the top right window. When the described object has been selected, its object ID is temporarily displayed at the mouse cursor (b). In a final step, the object ID needs to be entered in the bottom right text field (c).

As during Tasks 1 and 2, practice runs were performed prior to the recorded runs. The recorded attributes were elapsed times for each task as well as user interactions such as object selections and text inputs. Settings and models were varied such that no participant performed a task under same conditions twice.

\section{Study Results}

The results for all three tasks including the logged data and the participants' ratings and comments are included in this section.

\subsection{Task 1: Object-Text Correlations}

The elapsed times for each setting and model are visualized in the diagrams of Fig. 7 Altogether, the participants needed the most time to perform this task with Setting III (Mean: $77.92 \mathrm{sec}$, SD: 39.98) compared to Setting I (Mean: $39.99 \mathrm{sec}$, SD: 33.25) and Setting II (Mean: 58.55, SD: 34.61). Model 1 was the most complicated model (Mean: 69.59, SD: 37.48) compared to Model 2 (Mean: 47.68, SD: 24.25) and Model 3 (Mean: 59.20, SD: 49.06). Two-Way ANOVA (factors: model and setting) shows that there is a significant interaction between the respective factors $\left(F_{4,99, \gamma}=7.39, p-\right.$ Value $\left.<0.01\right)$. 

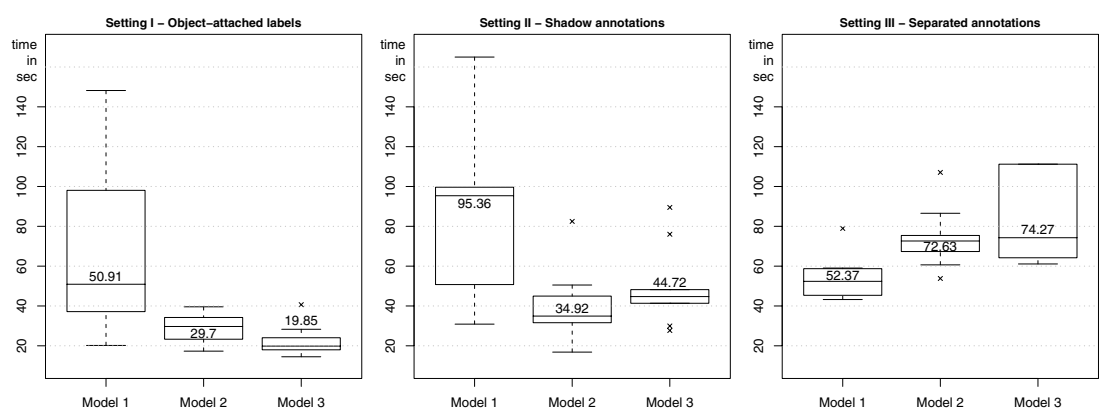

Fig. 7. Task 1: Elapsed times for Settings I-III and Models 1-3. The boxes indicate the lower and upper quartiles of the measured times as well as the Medians. In addition, smallest and largest values are shown together with outliers.

$F_{2,99, \alpha}=3.47$ (factor: type of model, $p-$ Value $=0.036$ ) indicates that the type of model has less effect on the time than the type of setting $\left(F_{2,99, \beta}=10.39, p-\right.$ Value $\left.<0.01\right)$.

After this first task had been performed, participants were asked to give a ranking of the individual settings. As can be seen in Table 1 (in Sect.5.3), $70 \%$ favored Setting I whereas $50 \%$ gave Setting III the worst rating.
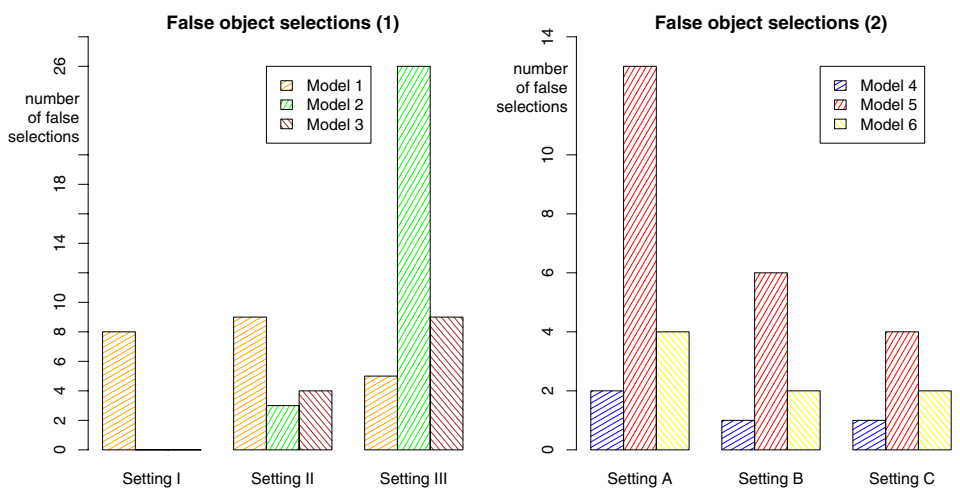

Fig. 8. Incorrectly selected scene objects

Figure 8(left) illustrates the number of incorrectly selected scene objects for each setting and model. The number of wrong selections varies for each setting depending on the model. In Settings I and II, Model 1 caused the most incorrect selections, whereas in Setting III participants had most problems with Model 2.

\subsection{Task 2: Object-Text Correlations with Additional Hints}

Again, user interactions and elapsed times were recorded. The diagrams in Fig. 9 show that participants performed this task fastest in Setting C for each model (Means/SDs: Setting A: 52.24/24.40, Setting B: 49.34/25.39, Setting C: 41.19/17.59). Model 5 was 
the most complicated model since participants needed the most time to perform the task with it (Means/SDs: Model 4: 40.0/15.88, Model 5: 62.23/28.28, Model 6: 40.51/15.29). Two-Way ANOVA (factors: model and setting) shows that there is no significant interaction between the respective factors $\left(F_{4,99, \gamma}=0.43, p-\right.$ Value $\left.=0.79\right)$. But the type of model has more influence than the type of setting $\left(F_{2,99, \alpha}=13.67, p-\right.$ Value $<0.01$ and $F_{2,99, \beta}=2.79, p-$ Value $=0.07$ ).
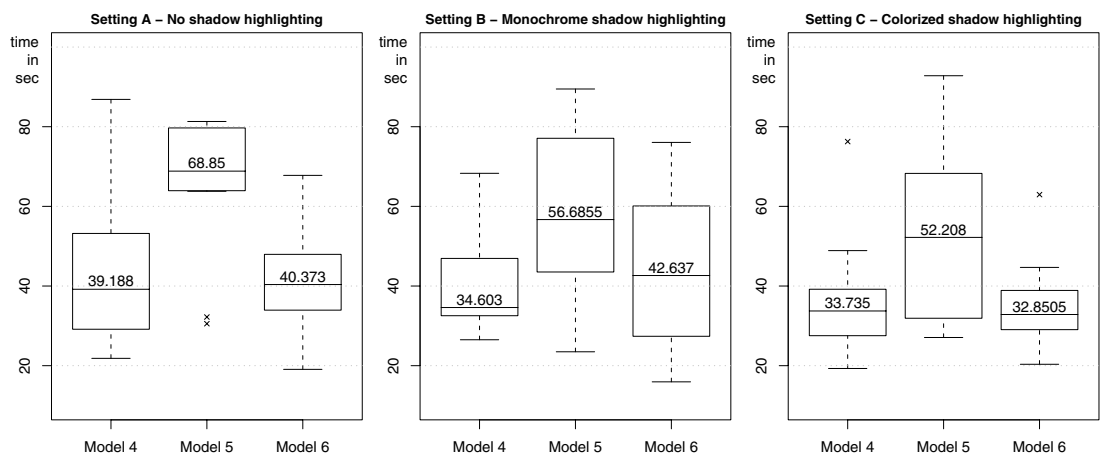

Fig. 9. Task 2: Elapsed times for Settings A-C and Models 4-6

The number of false object selections significantly decreased in Settings B and C, as is illustrated in Fig. 8 (right). Model 5 was the model that caused the most incorrect selections in all settings.

Participants used various techniques during this task, in which the search for objecttext correlations was supported by highlighted shadows. Some of them were quite fast in Setting C. When an annotation appeared, they compared scene object colors with the highlighted shadow color and quickly made their choice. However, other participants did not adapt their strategy to the type of setting. Some of them (14\%) even did not consider the highlighted shadows or stated that the shadows were not very helpful.

\subsection{Task 3: Task-Based Scene Exploration}

Task 3 was the most complex and time-consuming task. Since the settings were the same as in Task 1, we were primarily interested in a ranking. As can be seen in Table 1 it significantly differs to the ranking given after Task 1 . A clear majority of $97 \%$ rated Setting II to be the worst setting. Also, in contrast to the first rating, a majority of $64 \%$ favored Setting III. Furthermore, when participants were asked if they felt disturbed by annotations during scene exploration, nearly all said no.

The number of false object selections as well as incorrectly entered object IDs was marginal for each setting. But there were considerable elapsed time variations (see Fig. 10). Independently from the type of model, Setting II was the setting for which participants needed most time to perform this task (Means/SDs: Setting I: 98.35/25.06, Setting II: 176.00/43.08, Setting III: 93.89/24.37). Two-Way ANOVA yields that there is no significant interaction between the type of setting and the type of model $\left(F_{4,99, \gamma}\right.$ 
Table 1. Ratings for Settings I-III: after Task 1 (left) and Task 3 (right)

\begin{tabular}{|c|c|c|c|}
\hline Setting & 1st place & 2nd place & 3rd place \\
\hline \hline I & $70 \%$ & $22 \%$ & $8 \%$ \\
II & $11 \%$ & $47 \%$ & $42 \%$ \\
III & $19 \%$ & $31 \%$ & $50 \%$ \\
\hline
\end{tabular}

\begin{tabular}{|c|c|c|c|}
\hline Setting & 1st place & 2nd place & 3rd place \\
\hline \hline I & $36 \%$ & $64 \%$ & $0 \%$ \\
II & $0 \%$ & $3 \%$ & $97 \%$ \\
III & $64 \%$ & $33 \%$ & $3 \%$ \\
\hline
\end{tabular}
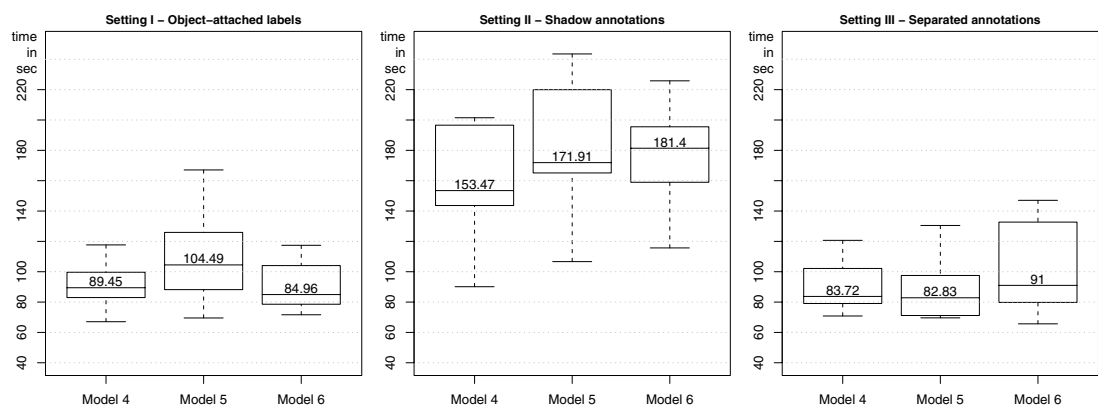

Fig. 10. Task 3: Elapsed times for Settings I-III and Models 4-6

$=0.78, p-$ Value $=0.54)$. But the type of setting had a significant influence on the measured time $\left(F_{2,99, \beta}=56.89, p-\right.$ Value $\left.<0.01\right)$ whereas the influence of the model's type was marginal $\left(F_{2,99, \alpha}=1.97, p-\right.$ Value $\left.=0.15\right)$.

\section{Discussion}

The purpose of this study was to increase our understanding about the assets and drawbacks of different techniques that integrate 3D data and text. We found that none of the evaluated techniques was perfect and that several factors had significant impact on how successfully certain tasks were completed. This included as expected the type of setting and it included the type of 3D model and the type of task.

\subsection{Task 1: Object-Text Correlations}

The diagrams in Fig. (7) show that participants needed most time to perform this first task with the setting-model pairs: Setting I - Model 1, Setting II - Model 1, and Setting III Model 3. To shed light on these observations, Figure 2 in which the individual models are depicted, can be analyzed. Model 1 is quite compact and the individual model components intersect with each other. Hence, using visual techniques to link between model component and text (as in Settings I and II) may not always be perfectly clear. Often, participants needed to ascertain their choices by time-consuming camera rotations. In contrast, Model 3 is more wide-spaced and composed of similarly shaped components. These visual features may make it more compatible with Settings I and II. However, Model 3 caused longest times in Setting III. This was probably due to descriptions such as "2nd biggest sphere" or "smallest cylinder" with which model components could 
be found. Even though the descriptions were clear, participants had problems evaluating the differences in size. False object selections (see Fig. 8 (left)) correlated with the elapsed times. The more time a participant needed to perform a particular task the higher the rate of false selections.

\subsection{Task 2: Object-Text Correlations with Additional Hints}

When the search for object-text correlations was combined with hints in the shadow, $75 \%$ of the participants stated explicitly that highlighted shadows facilitated the search. They first tried to find out where the annotation line ended before they ascertained their decision by including the shadow. However, some participants did not benefit from the highlighted shadows as much as we had expected. On one hand, the number of false object selections significantly decreased in Settings B and C, as is illustrated in Fig. 8 (right). On the other hand, the diagrams in Fig.9 show that participants performed this task significantly faster only in Setting C for each model. Since Setting B also provided hints in the shadow, we expected significant faster times here as well. It still has to be studied whether a longer practice period would yield other results.

\subsection{Task 3: Task-Based Scene Exploration}

This was a quite complex task. Basically, we aimed at provoking interferences between model components and annotations. As the rating in Table 1(right) and the diagrams in Fig. 10 show, Setting II was the worst setting for this task. There were two main reasons for this result: (1) the text did not always fit completely into the annotation shape causing participants to zoom into the scene to enlarge the text area and (2) annotations did often overlap.

Since the model render area and text were separated in Setting III, participants could not be disturbed by annotations during scene exploration. However, the measured times in Setting I and Setting III do not differ that much. This indicates that annotations, integrated into the 3D scene, have not such a high impact on a task-based scene exploration as we had expected. The post-questionnaire, in which participants were asked if they felt disturbed by annotations, confirmed these results. But nevertheless, as an observer, one often had an impression contrary to the participants' self-evaluations, especially in Setting I. One possible explanation might be that participants were so focused on searching for scene objects that they did not notice how much the annotations overlapped with the model. This aspect could be analyzed within another study.

\subsection{Design Suggestions}

After participants had performed the tasks, they were asked to itemize the features they would include if they were to design an information system. They could choose from a list of the techniques used during the study (multiple selections were possible) and they could make other suggestions. The results are: technique in Setting I: $83 \%$ of the participants would include this technique; technique in Setting II: $11 \%$; technique in Setting III: $89 \%$; and technique of highlighting objects in the shadow (Settings B and C): $69 \%$. In addition to these techniques, some participants suggested techniques such 
as using context menus (compare [6]) or displaying text only on explicit user request. These would be interesting suggestions to consider in a future study.

Furthermore, here are some comments participants gave for Settings I-III: Setting I "uses the most intuitive technique. It was helpful that the annotation size was adapted to the associated scene object's bounding box". The technique used in Setting II "was unaccustomed and requires practice. It was not suited for displaying text". In Setting III, "reading the scene object descriptions was time-consuming. Also, sometimes it was not clear which scene object was meant. But this setting was the most clearly arranged setting".

Our suggestions for the design of an information system, in which textual information is to be integrated, strongly depend on the application. When both short text labels and extensive texts are to be integrated, we suggest a combination of different techniques. The technique in Setting I seems to be suited for short labels. Using this technique scene objects referred to by annotations can be quite clearly identified. Also, there are no long distances between objects and annotations. However, "only" short labels because the use of this technique with longer texts may cause important parts of the scene to become occluded. An improvement of the object-line-text technique in Setting A (e.g., [10]) may be appropriate as well, although it is difficult to maintain an adequate scene layout during the scene exploration. For extensive texts, Setting III seems to be applicable because a user can explore a scene without any occlusions from the text. Furthermore, providing additional hints in the background can be helpful when object-text correlations need to be identified. The technique of highlighting the appropriate shadows (e.g., Settings B and C) could be a useful enrichment in an information system.

\section{Summary}

We have reported the results of a user study, in which we have examined three different techniques for providing textual information in interactive 3D illustrations. These techniques are: text labels directly attached to scene objects, texts located within a shadow plane, and textual information displayed in a window separated from the area in which the 3D model is rendered. Basically, we were interested in the effects when a 3D model and text are integrated into an appropriate layout and certain tasks are to be performed.

The study_-with thirty-six participants-had three tasks. For Task 1, participants were asked to correlate the displayed annotations with the model components to which the annotations referred. For Task 2, we were interested in the accessorial benefits when the additional hints of highlighted shadows are provided in the background. Finally, Task 3 aimed at text readability, text comprehension, and interferences between model components and text such as occlusions. All tasks required the interactive exploration of the 3D scene. As well as recording their actions, the participants were asked to answer questionnaires and to give further comments. As a result we found that none of the evaluated techniques was without drawbacks during all the tasks. But each technique has features that make it particularly applicable in certain situations. Based on our findings, we were able to point out some of those features.

For the future, we plan to investigate other techniques that give a user support during the navigation in interactive 3D illustrations. Unclear object-text correlations and scene occlusions are important issues in this regard. Concerning the design of a sec- 
ond study of that kind, it would be interesting to recruit participants who do not have any experience in the field of $3 \mathrm{D}$ computer graphics. Also, even though we had reasons for choosing arbitrary models, using real models may reveal results that can be more directly applied on specific interactive information systems.

\section{References}

1. Brinkley, J.F., Wong, B.A., Hinshaw, K.P., Rosse, C.: Design of an Anatomy Information System. IEEE Computer Graphics \& Applications 19 (1999) 38-48

2. Lok, S., Feiner, S.: A Survey of Automated Layout Techniques for Information Presentations. In: Proc. of the 1st International Symposium on Smart Graphics. (2001) 61-68

3. Fekete, J.D., Plaisant, C.: Excentric Labeling: Dynamic Neighborhood Labeling for Data Visualization. In: Proc. of the SIGCHI Conference on Human Factors in Computing Systems. (1998) 512-519

4. Golland, P., Kikinis, R., Halle, M., Umans, C., Grimson, W.E.L., Shenton, M.E., Richolt, J.A.: AnatomyBrowser: A Novel Approach to Visualization and Integration of Medical Information. Computer Aided Surgery 4 (1999) 129-143

5. Bell, B., Feiner, S., Höllerer, T.: View Management for Virtual and Augmented Reality. In: Proc. of UIST'01, ACM Symp. on User Interface Software and Technology. (2001) 101-110

6. Pommert, A., Höhne, K.H., Pflesser, B., Richter, E., Riemer, M., Schiemann, T., Schubert, R., Schumacher, U., Tiede, U.: Creating a High-Resolution Spatial/Symbolic Model of the Inner Organs Based on the Visible Human. Medical Image Analysis 5 (2001) 221-228

7. Schlechtweg, S., Strothotte, T.: Generating Scientific Illustrations in Electronic Books. In: Smart Graphics. Papers from the 2000 AAAI Spring Symposium. (2000) 8-15

8. Loughlin, M.M., Hughes, J.F.: An Annotation System for 3D Fluid Flow Visualization. In: Proc. of the Conference on Visualization '94. (1994) 273-279

9. Jung, T., Gross, M.D., Do, E.Y.L.: Annotating and Sketching on 3D Web Models. In: Proc. of the 7th International Conference on Intelligent User Interfaces (IUI 2002). (2002) 95-102

10. Ali, K., Hartmann, K., Strothotte, T.: Label Layout for Interactive 3D Illustrations. Journal of the WSCG (13th Int. Conf. in Central Europe on Computer Graphics, Visualization and Computer Vision) 13 (2005) 1-8

11. Herndon, K.P., Zeleznik, R.C., Robbins, D.C., Conner, D.B., Snibbe, S.S., van Dam, A.: Interactive Shadows. In: Proc. of ACM Symposium on User Interface Software and Technology. (1992) 1-6

12. Ritter, F., Sonnet, H., Hartmann, K., Strothotte, T.: Illustrative Shadows: Integrating 3D and 2D Information Displays. In: Proc. of the 8th International Conference on Intelligent User Interfaces (IUI 2003). (2003) 166-173

13. Chigona, W., Sonnet, H., Ritter, F., Strothotte, T.: Shadows with a Message. In: Proc. of the 3rd International Symposium on Smart Graphics. (2003) 91-101

14. Sonnet, H., Carpendale, M.S.T., Strothotte, T.: Integrating Expanding Annotations with a 3D Explosion Probe. In: Proc. of the Working Conference on Advanced Visual Interfaces (AVI 2004). (2004) 63-70 